\title{
Evidence for Initiation of Post-Zygotic Reproductive Isolation between Drosophila ananassae and D. pallidosa as Indicated by Reduction in the Fertility of Hybrid Males
}

\author{
Roshni Singh ${ }^{1} \&$ Bashisth Narayan Singh ${ }^{1}$ \\ ${ }^{1}$ Genetics Laboratory, Department of Zoology, Institute of Science, Banaras Hindu University, Varanasi, India \\ Correspondence: Bashisth Narayan Singh, Genetics Laboratory, Department of Zoology, Institute of Science, \\ Banaras Hindu University, Varanasi 221005, India. E-mail: bashisthsingh2004@rediffmail.com
}

Received: February 17, 2020

Accepted: March 10, $2020 \quad$ Online Published: March 30, 2020

doi:10.5539/ijb.v12n2p41

URL: doi: 10.5539/ijb.v12n2p41

\begin{abstract}
There are several barriers to preclude the gene flow between diverging populations. On the basis of their temporal nature, these can be broadly categorized into two forms: pre- and post-zygotic. Post-zygotic reproductive isolation can manifest in the form of reductions in hybrid fertility. Keeping this fact in view, in the present study, we studied sterility in hybrids of $D$. ananassae and D. pallidosa. Surprisingly a distinguishable pattern of infertility was found in the hybrids. This pattern, referred to as Haldane's rule, is often observed in hybrids of recently diverged populations or species. Reduction in the fertility of hybrids provides the clue of incipient kind of post-zygotic reproductive isolation in these two sibling species. This is the first report of hybrid sterility in this species pair. However, hybrid sterility is not very prominent especially when compared to that of other species pairs with the similar divergence time. Thus, on the basis of our results, we conclude that either sexual isolation between these sibling species is sufficient and does not require the aid of post-zygotic isolation to preclude gene flow or rate of divergence between $D$. ananassae and D. pallidosa is very slow in comparison to other species pair or even races of some species.
\end{abstract}

Keywords: D. ananassae, D. pallidosa, Haldane's Rule, Hybrid Male Sterility, Post-Zygotic Reproductive Isolation

\section{Introduction}

One of the most fundamental objectives in evolutionary biology is to understand the processes of species divergence. Yet, despite its importance, speciation has largely remained unexplored till date. Nevertheless much of our comprehension about speciation arises from a strong focus on reproductive isolation, because over the last decade, it has become clear that there is a parity between speciation and reproductive isolation, so that the origin of species" is largely synonymous to "the origin of reproductive isolation" (Dobzhansky, 1937; Mayr, 1942). Reproductive isolation or barriers to gene flow can be categorized by the temporal nature of their effect: pre-zygotic barriers occur before fertilization and post-zygotic barriers occur after fertilization (Coyne \& Orr, 2004). Post-zygotic reproductive isolation often manifest in the form of reductions in hybrid fertility (Hatfield \& Schluter, 1999). Hybrid sterility can eventually be attributed to Dobzhansky-Muller incompatibilities (Hutter, 1997) which typically evolves when diverging populations accumulate different alleles at two or more loci and these are incompatible when brought together in hybrid genomes. Therefore, negative epistasis between these alleles render hybrids inviable or sterile (Bateson, 1909; Dobzhansky, 1937; Muller, 1942). A clear understanding of hybrid sterility thus requires a clear understanding of Dobzhansky-Muller incompatibilities. Hence, both hybrid incompatibilities and species-specific differences play a vital role in the development of post-zygotic reproductive isolation between incipient species. Thus, identification of hybrid incompatibilities measured as increased sterility of hybrids is a major goal in evolutionary biology. Therefore, study of hybrids by laboratory hybridization is a crucial step in resolving the evolution of post-zygotic isolation as well as speciation.

In the present study, we focused on the hybrid sterility between $D$. ananassae and $D$. pallidosa as they can be hybridized in the laboratory. The species pair is unique due to the presence of strong sexual isolation in sympatric situation but absence of post mating barriers such as hybrid inviability or sterility in the interspecific hybrids (Oguma, 1993; Sawamura et al., 2008). While a number of studies have focused on the premating isolation in these 
sibling species (Futch, 1966, 1973; Vishalakshi \& Singh, 2006) but studies on post-zygotic isolation has been somewhat neglected. However, post-zygotic isolation has been extensively studied in many other Drosophila species (Cabot et al., 1994; Davis et al., 1994; Perez \& Wu, 1995; Tao, Xhen, Hartl, \& Laurie, 2003; Sweigart, 2010). It is surprising that evolutionary biologists have not identified any post-zygotic isolation between these sibling species, not even asymmetrical sterility in hybrid sons (Haldane, 1922), which is an early sign of post-zygotic isolation. Therefore, we were provoked to identify post-zygotic isolation in these sibling species if any. Keeping the above in view, we investigated the fertility of hybrids (hybrid sterility) between $D$. ananassae and $D$. pallidosa and compared with the parental species.

\section{Materials and Methods}

\subsection{Drosophila Stocks}

One mass culture stock of $D$. ananassae, established from flies collected from Pondicherry (PC) India was employed in the present study. One wild type strain of D. pallidosa was used. This strain is NOU 88 which was kindly provided by Prof. M. Matsuda of Kyorin University, Japan. These stocks are being maintained in the laboratory on the simple yeast agar culture medium at approximately $24^{\circ} \mathrm{C}$ following 12 hours' light and dark cycle.

\subsection{Fertility Assay}

For the fertility assay, we checked the larval activity. This procedure was earlier used by Kim and Watanabe (1989) and Banerjee and Singh (2016). All the experiments of fertility assay were categorised into two groups that was group I and group II. Group I comprises homogamic and heterogamic crosses of parental species. Group II comprises all the crosses of reciprocal hybrid daughters and sons with parental species and hybrid itself.

\subsubsection{Experimental Design to Assay Fertility in Group I}

20 pairs of 8 days old females and males from each strain were transferred to the bottles to collect virgins. Further, after the emergence of new flies virgin females and males were sorted under stereobinocular microscope on the basis of the presence of the ovipositor and aged for 8 days in food vials. After the completion of 8 days,

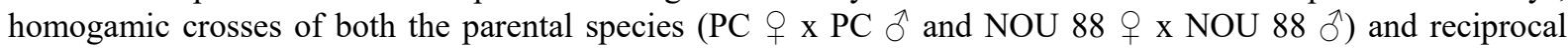
heterogamic crosses between $D$. ananassae PC and D. pallidosa NOU 88 (PC $q$ x NOU 88 $\overbrace{}^{\lambda}$ and NOU 88 × 9 PC ${ }^{1}$ ) were set. Each cross was set in 20 vials by keeping single female with single male (1 pair/vial) (Figure 1).

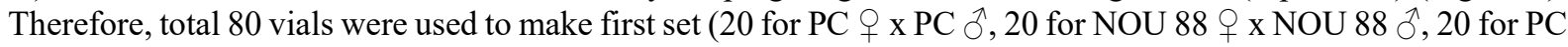

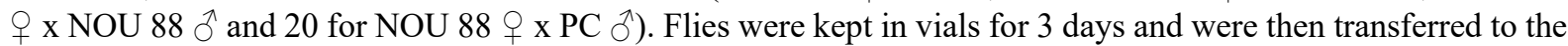
fresh food vials from first sets of vials to set second set. Similarly, third and fourth sets were also set by transferring flies from second and third sets of vials respectively into fresh vials after each 3 days. Those vials where females and males were not alive or flew during transfer were excluded from the experiments. After about 10 days, the first sets of vials were inspected for larval activity. Vials in which larval activity was found were recorded as having a fertile female. The vials in which no larval activity was seen were kept for few more days and regularly checked for larval activity. The corresponding second, third and fourth sets of vials were also inspected for larval activity. Vials in which no larval activity was noted in all the four sets of vials were noted as having sterile female. Hence, the number of fertile and sterile females from each cross was scored.

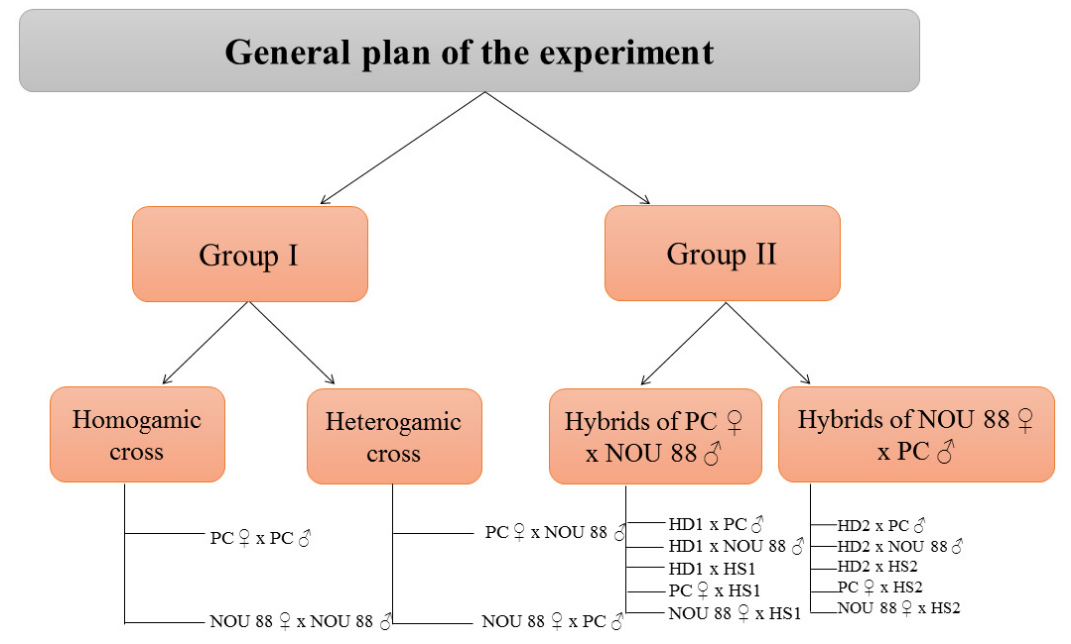

Figure 1. Schematic presentation of the experiments 


\subsubsection{Experimental Design to Assay Fertility in Group II}

20 pairs (females and males in equal number) of 8 days old females and males from each strain were transferred to the bottles to collect virgin. Further, after the emergence of new flies virgin females and males were sorted under stereobinocular microscope on the basis of the presence of the ovipositor and aged for 8 days in food vials. After the completion of 8 days, reciprocal heterogamic crosses between D. ananassae PC and D. pallidosa NOU 88 (PC

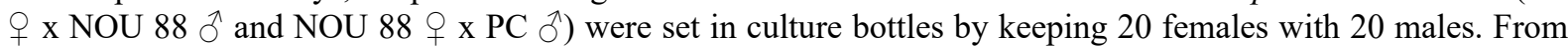
these bottles hybrid daughters and hybrid sons (Daughters of PC $q$ x NOU 88 خ (HD1), sons of PC $q$ x NOU $88 \lambda$

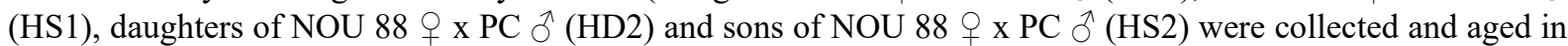
food vials for 8 days which were further used to set the experimental vials. From the hybrids of PC $q \times$ NOU $88 \AA$ cross, five sets of crosses were set with parental males, females and hybrid themselves: HD1 x PC $\hat{\partial}$, HD1 x NOU $88 \hat{\jmath}$, HD1 x HS1, PC $q$ x HS1, NOU 88 q $\times$ HS1 (Figure 1). Similarly from the hybrids of NOU 88 q $\times$ PC $\delta$ cross, five sets of crosses were also set with parental males, females, and hybrid themselves: HD2 x PC $\hat{\jmath}, \mathrm{HD} 2 \mathrm{x}$

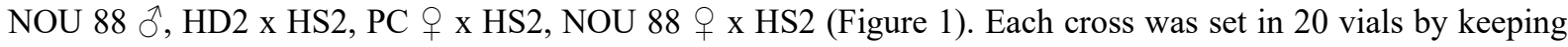
single female with single male (1 pair/vial). Flies were kept in vials for 3 days and were then transferred to the fresh food vials from first sets of vials to set second set. Similarly, third and fourth sets were also set by transferring flies from second and third sets of vials respectively into fresh vials after each 3 days. Those vials where females and males were not alive or flew away during the transfer were excluded from the experiments. After about 10 days, the first sets of vials were inspected for larval activity. Vials in which larval activity was found were recorded as having a fertile female. The vials in which no larval activity was seen were kept for few more days and regularly checked for larval activity. The corresponding second, third and fourth sets of vials were also inspected for larval activity. Vials in which no larval activity was noted in all the four set of vials were noted as having sterile female. Hence, the number of fertile and sterile females from each cross was scored.

\subsection{Staining of Testis}

To study the individualization of spermatids, testis was stained by using the method of Maside, Barrel, \& Naveira (1998) with slight modification in stain. Testis of reproductively mature (8-10 days old) males (both parental species as well as both the reciprocal hybrid sons) were dissected out without disturbing the integrity of testis on a slide containing few drops of insect saline solution $(0.67 \% \mathrm{NaCl})$. Further, they were treated with a fixing solution ( $45 \%$ acetic acid) for a very short period $(10 \mathrm{sec})$ and immediately transferred to a new slide with few drops of $2 \%$ lacto-aceto-orcein stain and kept for 20-25 minutes. They were then washed in $45 \%$ acetic acid to remove extra stain. Gently a coverslip was placed over the testis and excess of stain were removed with the help of blotting paper. Prepared slides were then observed under a phase contrast microscope (Nikon Eclipse 800) at 10X magnification.

\subsection{Protein Estimation}

Protein estimation of reproductively mature (8-10 days old) male's testis of parental species as well as both the reciprocal hybrid sons were done by using Bradford method (1976).

\subsection{Statistical Analysis}

Chi-square values were calculated from $\mathrm{RxC}$ contingency table to check whether there are significant differences in number of fertile and sterile flies between homogamic and heterogamic crosses $(2 \times 2)$, between different crosses of both the reciprocal hybrids $(2 \times 2)$ and between different crosses of hybrids and parental species $(4 \times 2)$.

\subsection{Calculations of Different Isolating Barriers}

We calculated different isolating barriers by using our data to know the strength of different isolating barriers between these two sibling species (Ramsey, Bradshaw, \& Schemske, 2003; Bono \& Markow, 2009).

\subsubsection{Post-Mating-Prezygotic Isolation}

$\mathrm{RI}_{\mathrm{PMPZ}}=1$-fertility of heterospecific crosses/fertility of homospecific crosses

\subsubsection{Hybrid Sterility}

$\mathrm{RI}_{\mathrm{HS}}=$ (1-proportion fertile hybrid males/proportion fertile pure males)/2

\subsubsection{Total Reproductive Isolation}

We calculated the absolute contribution (AC) of each stage (n) of reproductive isolation (RI) to total isolation using equation 4 from Ramsey et al. (2003). 


$$
\mathrm{AC}_{n}=\mathrm{R} I_{n}\left(1-\sum_{i=1}^{n-1} A C_{i}\right)
$$

Further, we calculated total isolation (T) using equation 5 (Ramsey et al., 2003).

$$
\mathrm{T}=\sum_{i=1}^{m} \mathrm{~A} C_{i}
$$

Values can range between 0 , indicating no isolation, and 1, indicating complete reproductive isolation.

\section{Results}

Table 1. RxC contingency chi-square tests to check the differences in fertility between homogamic and heterogamic crosses, between different crosses of both the reciprocal hybrids and between different crosses of

\begin{tabular}{|c|c|c|c|c|c|}
\hline Type of crosses & $\mathrm{n}$ & No. of fertile flies & No. of sterile flies & Chi-square & \\
\hline $\overrightarrow{\mathrm{PC}}+9 \mathrm{PC} 0^{n}$ & 18 & 18 & 0 & & \\
\hline NOU 88 9 x NOU 88 ـ & 15 & 14 & 1 & & \\
\hline PC 9 x NOU $880^{-1}$ & 17 & 14 & 3 & & -6.83 \\
\hline NOU $88+9$ × PC 0 & 20 & 20 & 0 & & \\
\hline HD1 x PC 0 & 17 & 15 & 2 & & \\
\hline $\mathrm{HD} 2 \times \mathrm{PC} 3$ & 19 & 18 & 1 & & 2.23 \\
\hline $\mathrm{PC}+9 \times \mathrm{PC} \hat{0}^{-1}$ & 18 & 18 & 0 & & \\
\hline NOU 88 ㅇ x NOU 88 今 & 15 & 14 & 1 & & \\
\hline HD1 x NOU 88 त & 17 & 11 & 6 & & \\
\hline HD2 x NOU 88 त & 17 & 16 & 1 & & \\
\hline $\mathrm{PC} q \times \mathrm{PC} 0^{\pi}$ & 18 & 18 & 0 & & $-12.24 * *$ \\
\hline NOU 88 × 9 NOU $88 \overbrace{}^{\lambda}$ & 15 & 14 & 1 & & \\
\hline HD1 x HS1 & 17 & 14 & 3 & & \\
\hline $\mathrm{HD} 2 \times \mathrm{HS} 2$ & 20 & 17 & 3 & & \\
\hline $\mathrm{PC}+9 \times \mathrm{PC} 0$ & 18 & 18 & 0 & & 3.84 \\
\hline NOU 88 + x NOU 88 ـ & 15 & 14 & 1 & & \\
\hline $\mathrm{PC}$ ㅇ $\mathrm{x} \mathrm{HS} 1$ & 17 & 9 & 8 & & \\
\hline PC $q$ x HS2 & 20 & 14 & 6 & & $1436 * *$ \\
\hline $\mathrm{PC}+9 \times \mathrm{PC} 0^{\pi}$ & 18 & 18 & 0 & & \\
\hline NOU 889 x NOU 88 ـ & 15 & 14 & 1 & & \\
\hline NOU $88+9$ x HS1 & 17 & 13 & 4 & & \\
\hline NOU 88 우 $\mathrm{HS} 2$ & 17 & 17 & 0 & $7.71^{* *}$ & \\
\hline $\mathrm{PC}+9 \times \mathrm{PC} 0^{\pi}$ & 18 & 18 & 0 & & $-9.17^{*}$ \\
\hline NOU 88 x NOU 88 ـ & 15 & 14 & 1 & & \\
\hline
\end{tabular}
hybrids and parental species

${ }^{* *} p<0.001,{ }^{*} p<0.01$.

PC, D. ananassae PC; NOU 88, D. pallidosa NOU 88; HD1, hybrid daughters of $D$. ananassae PC $q$ x $D$.

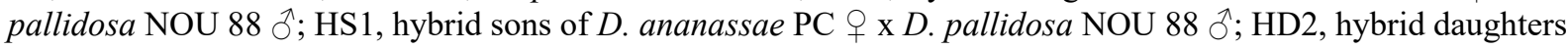

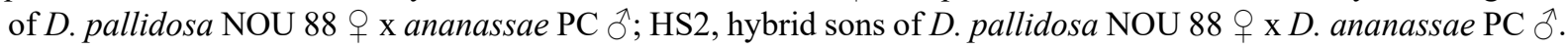

It is clear that females mated with conspecific males do not show significantly more fertility than females mated with a heterospecific males (Table 1). Therefore, fertility between homogamic and heterogamic crosses are statistically not significant and occurrence of similar fertility levels in conspecific and heterospecific matings indicate a lack of postmating prezygotic isolation in these sibling species pair. In contrast, when D. ananassae PC

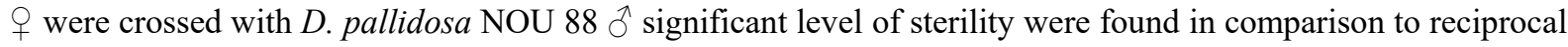
cross $(\chi 2=5.71, p<0.01)$ because $D$. ananassae PC females were less fertile with $D$. pallidosa NOU 88 males than conspecific males. Therefore, among heterogamic crosses, D. pallidosa NOU 88 females mated more often with $D$. ananassae PC males than $D$. ananassae PC females mated with $D$. pallidosa NOU 88 males. No significant differences were found in the number of fertile and sterile flies between HD1 x PC and HD2 x PC, HD1 x HS1 and 
HD2 $x$ HS2 $\left(\chi^{2}=1.10, \chi^{2}=1.66\right.$ respectively). The numbers of fertile and sterile flies were not found to differ significantly in the crosses of both the hybrid daughters with $D$. ananassae PC, both the hybrid daughters with hybrid sons and heterospecific crosses in comparison to the parental species $\left(\chi^{2}=2.23, \chi^{2}=3.84, \chi^{2}=6.83\right)$. Whereas drastically reduced fertility was found in the crosses of both the hybrid daughters with $D$. pallidosa NOU $88\left(\chi_{2}=12.24, p<0.001\right)$, both the hybrid sons with $D$. ananassae PC $(\chi 2=14.36, p<0.001)$ as well as $D$. pallidosa NOU $88\left(\chi^{2}=9.17, p<0.01\right)$ in comparison to parental species. Significant differences were found in the fertile and sterile flies between crosses of both the reciprocal hybrid daughters with D. pallidosa NOU $88\left(\chi^{2}=\right.$ $9.40, p<0.001)$, crosses of both the reciprocal hybrid sons with $D$. ananassae $\mathrm{PC}(\chi 2=7.51, p<0.001)$ as well as $D$. pallidosa NOU $88(\chi 2=7.71, p<0.001)$. Between HD1 x NOU 88 and HD2 x NOU 88 , HD1 x NOU 88 cross was more severe in terms of sterility because more number of flies were found to be sterile in this respective cross in comparison to other one. Between PC $q$ x HS1 and PC $q$ x HS2, PC $q$ x HS1 was more critical in terms of sterility because more number of flies were sterile in this cross in comparison to other one. Between NOU 88 9 $\mathrm{x}$ HS1 and NOU $88 q \times$ HS2, NOU $88 q \times$ HS1 is more severe in comparison to other. Therefore, hybrid sons (HS1) obtained by crossing the $D$. ananassae $\mathrm{PC}$ + with $D$. pallidosa NOU 88 oxhibited high level of reduction in the fertility with both the parental species rather than HS2 and hybrid sons of both the reciprocal cross exhibiting more sterility with ancestral species (D. ananassae PC).

Maximum sterility was found in the cross of hybrid sons with parental species $(25.35 \%)$ in comparison to the cross of hybrid daughters with parental species (14.28\%) and hybrid daughters with hybrid sons $(14.28 \%$ ) (Figure 2).Value of $\mathrm{RI}_{\mathrm{PMPZ}}$ was -0.05 , indicating that no post-mating-prezygotic isolation was found between $D$. ananassae and D. pallidosa. Hybrid sterility for HS1 was 0.17 whereas for HS2, it was 0.065 clearly indicating that HS1 are more sterile in comparison to HS2 (Table 2). Total reproductive isolation for HS1 was high rather than HS2 (Table 2). We did not find any atrophy in the testis of both the reciprocal hybrid sons in comparison to parental species which show that testis of parental species as well as both the reciprocal hybrid sons were fully normal and dense (Figure 3A, 3B, 3C, 3D). Protein in the testis of HS1 was significantly less in comparison to parental species as well as HS2 (Figure 4), indicating the severity of HS1.

Table 2. Strength and absolute contribution of different kinds of reproductive isolating barriers of both the reciprocal crosses as well as both the hybrid sons

\begin{tabular}{lllll}
\hline & \multicolumn{2}{l}{ PC x NOU 88 } & \multicolumn{2}{l}{ NOU 88 x PC } \\
Type of isolating barrier & Strength & Absolute contribution & Strength & Absolute contribution \\
\hline PMPZ & 0.565 & 0.565 & 0.375 & 0.375 \\
Hybrid sterility (HS 1 and HS 2) & 0.170 & 0.074 & 0.065 & 0.024 \\
Hybrid inviability (HS 1 and HS 2) & 0.000 & 0.000 & 0.000 & 0.000 \\
Total isolation & 0.637 & 0.266 & 0.399 & 0.264 \\
\hline
\end{tabular}

Note: In both the crosses, maternal strains were written first.

PC, D. ananassae PC; NOU 88, D. pallidosa NOU 88; HS1, hybrid sons of $D$. ananassae $\mathrm{PC}$ × $\mathrm{x} D$. pallidosa NOU 88 o ; HS2, hybrid sons of $D$. pallidosa NOU 88 q x D. ananassae PC $\widehat{o}$.

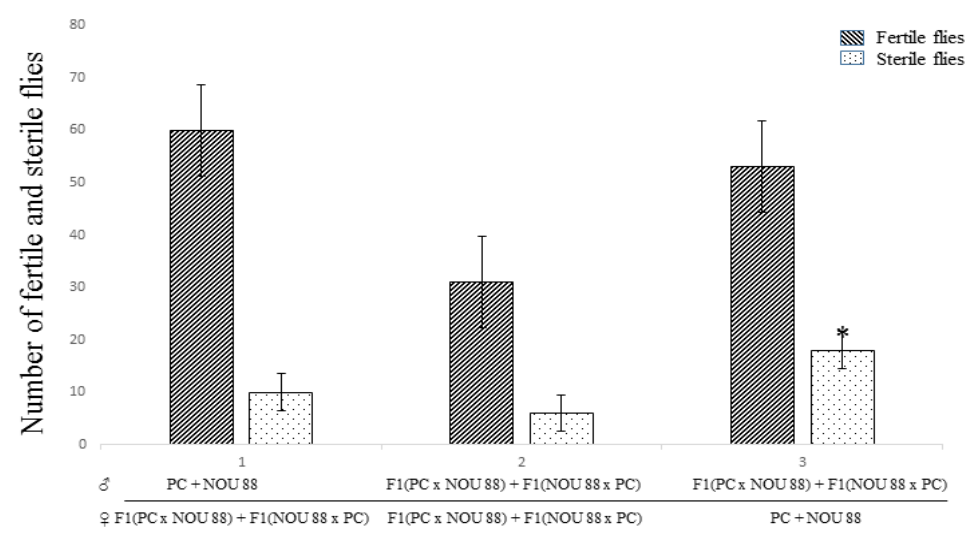

Figure 2. Fertile and sterile flies in crosses of hybrid daughters, hybrid sons with parental species and hybrid daughters with hybrid sons $\left({ }^{*} p<0.001\right)$ 
A

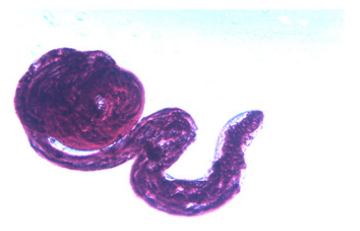

$\underline{200 \mu \mathrm{m}}$

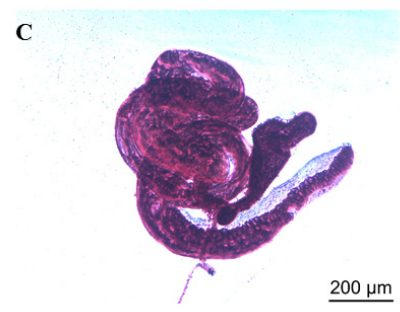

B

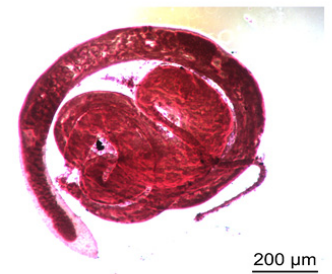

D

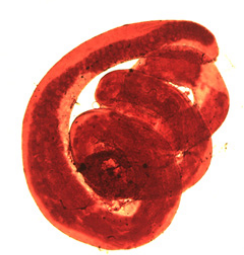

$\underline{200 \mu \mathrm{m}}$

Figure 3. Photographs showing morphology of the testis (A) D. ananasaae (B) D. pallidosa (C) Hybrid sons of $D$.

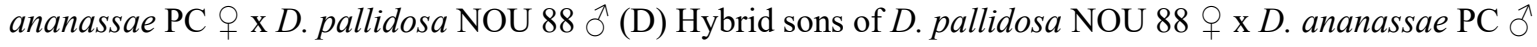

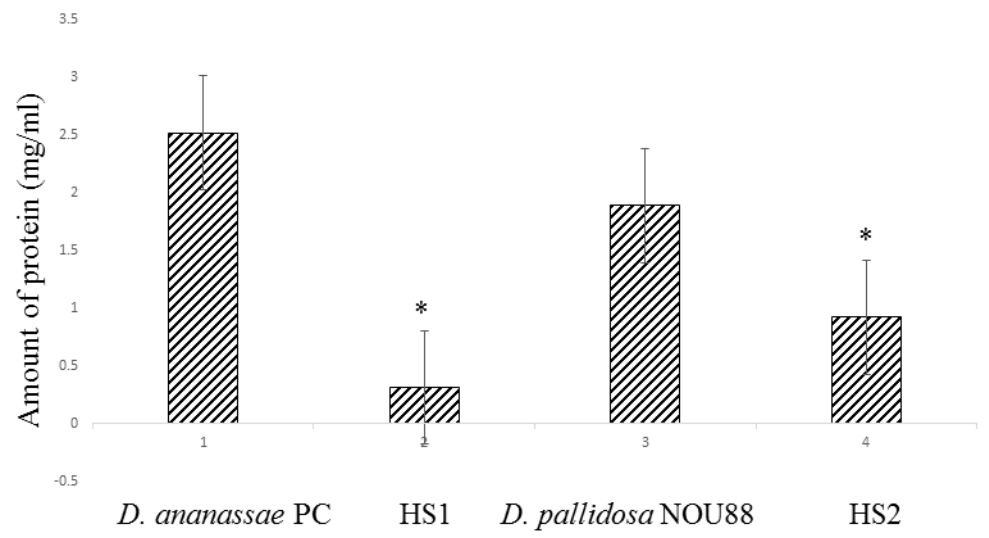

Figure 4. Amount of protein present in the testis of both the parental species as well as both the reciprocal hybrids $(* p<0.001)$

\section{Discussion}

In a long history of evolutionary biology, hybrid male sterility was studied extensively by using Drosophila as a model because Drosophila is particularly well suited to study such kind of reproductive barriers, as species within this genus are highly variable in degree of reproductive isolation from noninterbreeding species to hybridizing species (Yukilevich \& True, 2008; Civetta \& Gaudreau, 2015; Brill, Kang, K. Michalak, P. Michalak, \& Price, 2016). Therefore, in the present study, we studied hybrid sterility between these two sibling species to understand the exact status of post-zygotic isolation by means of larval activity. We found that when parental females mate with F1 males, no larval activity was found in some vials and the lack of larval activity indicate the failure of hybrid sons as well as parental species. However, this is the first report of the significant reduction in fertility of hybrid sons between $D$. ananassae and $D$. pallidosa which is very surprising. Earlier it was known that hybrids are fully fertile and normal (Oguma, 1993; Sawamura et al., 2008). In many eukaryotic organisms, especially Drosophila, hybrid male sterility is a key event of post-zygotic reproductive isolating mechanism, and, our results of experimental hybridization between these two sibling species provides the evidence of preliminary post-zygotic reproductive isolation. In the present study, post-zygotic isolation might be the result of distorted interaction of genetic combination of the parental lineages that, although functional in their normal genetic backgrounds, reduce fitness when recombined in hybrids (Rundle, Nagel, Boughman, \& Schluter, 2000; Burton, Ellison, \& Harrison, 2006; Rogers \& Bernatchez, 2006; Gow, Peichel, \& Taylor, 2007). Several genes are involved in the expression of this complex and polygenic trait (Gomes \& Civetta, 2015). Three genes or more were identified in hybrid sterility 
of Drosophila simulans (Johnson, 2000), whereas Wu, Johnson, \& Palopoli (1996), found over 100 genes on the X chromosome, contributing to hybrid male sterility as well as many genes reside on the autosomes (Tao et al., 2003; Araripe, Montenegro, Lemos, \& Hartl, 2010; Dickman \& Moehring, 2013). In addition to extensive X and autosomal studies, a number of spermatogenesis genes that is differentially expressed between hybrids and parental species, were revealed in myriad of Drosophila species by the gene expression studies (Porter \& Johnson, 2002; Michalak \& Noor, 2003, 2004; Johnson \& Porter, 2007; Landry, Hartl, \& Ranz, 2007; Moehring, Teeter, \& Noor, 2007).

Our results were consistent with the Haldane's rule which states that the heterogametic sex will be affected first during the course of evolution as well as speciation. There are three main theories to explain the Haldane's rule: The faster-X theory, the faster-male theory (Meiklejohn, Parsch, Ranz, \& Hartl, 2003; Zhang, Hambuch, \& Parsch, 2004; Eads, Colbourne, Bohuski, \& Andrews, 2007; Malone \& Michalak, 2008; Schilthuizen, Giesbers, \& Beukeboom, 2011) and the dominance theory (Turelli \& Orr, 1995). The basis of all three theories are same, that is epistatic genes are co-adapted within species but not between species. Thus, as a consequence of hybridization, these co-adapted genes are replaced by gene variants. Due to the result of this replacement, old combinations will break and new combinations will form which are incompatible. However, we discuss our results in the light of faster-X theory. According to faster-X theory, genes on the sex chromosomes of diploid organisms evolve faster in comparison to genes on autosomes. Epistatic interactions of genes on a fast-evolving chromosome are more prone for disruption when brought into the foreign genetic background than genes on slowly evolving autosomes, due to their larger divergence. Therefore, corresponding to the faster- X theory, we found that, out of two hybrid sons of both the reciprocal crosses, hybrid sons (HS1) obtained by crossing D. ananassae $\mathrm{PC} q$ with D. pallidosa NOU 88 $\delta$ exhibit highly reduced fertility with both the parental species rather than reciprocal hybrid sons (HS2). It might be possible that due to the faster evolution of X chromosome, D. ananassae's X chromosome might have diverged much more, as $D$. ananassae were considered as an ancestral species (Futch, 1966). Thus, being more diverged, $\mathrm{X}$ chromosome of $D$. ananassae is more incompatible with the $\mathrm{Y}$ chromosome of $D$. pallidosa in HS1 in comparison to $\mathrm{X}$ chromosome of $D$. pallidosa with the $\mathrm{Y}$ chromosome of $D$. ananassae in HS2. This faster evolution can not only enhance genetic divergence for X-linked genes but also create X-autosome incompatibilities as well as X-Y incompatibilities in hybrids. There are several studies (Good, Dean, \& Nachman, 2008; Kitano et al., 2009; Garrigan, Kingan, Geneva, Vedanayagam, \& Presgraves, 2014) which reported the large effect of sex chromosome on hybrid sterility suggesting that hybrid male sterility is frequently caused by incompatibilities between the $X$ and $Y$ chromosomes (Mishra \& Singh, 2007) or between the $X$ and heterospecific autosomal alleles and it might be possible that due to this incompatibilities between the $\mathrm{X}$ and $\mathrm{Y}$ chromosomes or between the $\mathrm{X}$ and heterospecific autosomal alleles, hybrid sons are first to suffer malfunctioning. Hybrids also might have defects in biochemical or physiological systems that also impair an organism's ability to perform complex functions as we found significant variations in protein of testis indicating its important role in sterility of hybrid males between these two sibling species. This was also consistent with the fact that proteins in the reproductive tissues diverge more rapidly than nonreproductive tissues (Ranz, Castillo-Davis, Meiklejohn, \& Hartl, 2003; Zhang, Sturgill, Parisi, Kumar, \& Oliver, 2007; Assis, Zhou, \& Bachtrog, 2012; Harrison et al., 2015). These proteins tend to evolve rapidly by positive selection between closely related species (Haerty et al., 2007). Thus, in future, studies related to microarray of hybrid's testis will answer lots of questions related to these species pair and uncover the mystery of these species pair. Second, hybrids may exhibit intermediate or mixed courtship behaviours that reduce mating success with both parent species. Third, hybrid courtship could be disrupted by negative epistatic interactions between courtship-specific genes. Hybrid behavioural sterility occurs when hybrids are unable to perform courtship behaviours (Davies, Aiello, Mallet, Pomiankoski, \& Silberglide, 1997; Noor, 1997; Coyne, Elwyn, Kim, \& Llopart, 2004). Further, we predict that there might be the effect of cytoplasm (that is, mitochondrial background) on sterility also, as different patterns were evidenced in the reciprocal crosses, where effect of cytoplasm is strong with the older species (Niehuis, Judson, \& Gadau, 2008; Oliveira, Raychoudhury, Lavrov, \& Werren, 2008; Werren et al., 2010).

In addition to hybrids, sex of the parental species also influences the fertility of hybrids. First, if only hybrids were responsible for the reduction in the fertility then reduction in the fertility should also found where both the partners were hybrids, but we did not find any significant reduction in the fertility of hybrids with hybrids themselves whereas hybrids with both the parental species were showing reduction the fertility. Second, results differ with both the parents, reciprocal hybrid sons were showing high reduction in the fertility with $D$. ananassae $\mathrm{PC} q$ in comparison to $D$. pallidosa NOU 88 , indicating that $D$. ananassae, as a partner were choosier rather than $D$. pallidosa against the hybrid males, as $D$. ananassae is an ancestral species in this species pair. Thus, being an ancestral species, $D$. ananassae retained very rigid mate recognition system and because of having rigid mate recognition system, females from the ancestral lineage discriminate more against hybrid males, due to the loss of 
male mating components associated with incompatibilities between the $\mathrm{X}$ and $\mathrm{Y}$ chromosomes or between the $\mathrm{X}$ and heterospecific autosomal alleles (Kaneshiro, 1983). Therefore, strict discriminative behaviour of parental species specially, ancestral species, due to rigid mate recognition system implicate synergistic effect in association of X-Y or X-autosome incompatibilities in the reduction of fertility of hybrids.

Occurrence of reduction of fertility of hybrid males, also, might be due to the atrophies in the testis morphology as morphological abnormalities of the testis is one of the important phenotypic measures of sterility (Civetta \& Gaudreau, 2015; Gomes \& Civetta, 2015). Sundararajan and Civetta (2011) reported significant under expression of few genes localized to testis in sterile hybrids relative to parental species. In contrast to this, we did not find any obvious differences in testis's morphology of sterile F1 males. However, the physiological and developmental processes that are involved in testis formation have diverged to such an extent that the two systems have become incompatible within $\sim 0.7$ million years. Whereas pairwise divergence time for $D$. ananassae and $D$. pallidosa is 1.68 MYA (Russo, Beatriz, Frazão, \& Voloch, 2013). Therefore, despite of 1.68 MYA of divergence, lack of morphological abnormalities in hybrid male's testis is quite surprising. At present we can only say that the differences at the level of morphology might be the last target of evolution. It might be possible that detailed electron microscopy analysis of parental species and hybrids would identify any minor morphological anomalies in the testis of males in near future.

We found reduction in fertility of hybrid sons rather than complete sterility of hybrid sons between $D$. ananassae and $D$. pallidosa, suggesting that post-zygotic isolation has just begun to arise. Therefore, our study provides very early sign of post-zygotic reproductive isolation. However, in D. melanogaster group, even closely related species like $D$. sechellia and $D$. mauritiana carry many incompatibilities which is responsible for hybrid male sterility (Masly \& Presgraves, 2007). In crosses between D. melanogaster and D. simulans, all F1 hybrids are dead, as it is known that the situation is more severe in older species pairs (Shapiro et al., 2007).

\section{Conclusion}

On the basis of our results, we can speculate that this species pair is unique in comparison to other species pairs. Surprisingly, sexual isolation without post-zygotic isolation was sufficient to preclude gene flow (Bock \& Wheeler, 1972) between these sibling species. Whereas, Kao, Zubair, Salomon, Nuzhdin, \& Campo (2015) reported post-zygotic isolation between United States and Caribbean strains of Drosophila melanogaster. Therefore, it is questionable that how sexual isolation could be the signature of speciation or divergence between these sibling species while strong sexual isolation occurs even among strains. As, we have found greater degree of intraspecific sexual isolation in $D$. pallidosa in comparison to interspecific isolation between $D$. ananassae and $D$. pallidosa (Singh and Singh unpublished). It may be possible that rate of divergence between $D$. ananassae and $D$. pallidosa is very slow in comparison to other species pair or even races of some species. Therefore, if the rate of divergence is slow between them in comparison to races or strains and races or strains have not achieved full status of species. How have D. ananassae and D. pallidosa been assigned distinct species status?

\section{Acknowledgements}

We thank Prof. M. Matsuda, Kyorin University, Tokyo, Japan, for providing the stocks of $D$. pallidosa. Financial support in the form of UGC Research Fellowship and CSIR JRF New Delhi to RS is gratefully acknowledged. We thank the anonymous reviewer for valuable suggestions.

\section{Conflict of interests}

The authors declare that there is no conflict of interests regarding the publication of this paper.

\section{References}

Araripe, L. O., Montenegro, H., Lemos, B., \& Hartl, D. L. (2010). Fine-scale genetic mapping of a hybrid sterility factor between Drosophila simulans and D. mauritiana: The varied and elusive functions of "speciation genes". BMC Evolutionary Biology, 10, 385-397. doi: 10.1186/1471-2148-10-385

Assis, R., Zhou, Q., \& Bachtrog, D. (2012). Sex-biased transcriptome evolution in Drosophila. Genome Biology and Evolution, 4, 1189-1200. doi: 10.1093/gbe/evs093

Banerjee, P., \& Singh, B. N. (2016). The Drosophila bipectinata species complex: Degree of sterility and dystrophied ovaries in interspecific hybrid females. Journal of Genetics, 95, 167-170. doi: 10.1007/s12041-015-0600-3

Bateson, W. (1909). Heredity and variation in modern lights. In A. C. Steward (Ed.), Darwin and modern science (pp. 85-101). Cambridge, UK: Cambridge University Press. 
Bono, J., \& Markow, T. A. (2009). Postzygotic isolation in cactophilic Drosophila: Larval viability and life-history traits of D. mojavensis/D. arizonae hybrids. Journal of Evolutionary Biology, 22, 1387-1395. doi: 10.1111/j.1420-9101.2009.01753.x

Bradford, M. M. (1976). A rapid and sensitive method for the quantitation of microgram quantities of protein utilizing the principle of protein-dye binding. Analytical Biochemistry, 72, 248-254. doi: 10.1016/0003-2697(76)90527-3

Brill, E., Kang, L., Michalak, K., Michalak, P., \& Price, D. K. (2016). Hybrid sterility and evolution in Hawaiian Drosophila: Differential gene and allele-specific expression analysis of backcross males. Heredity, 117, 1-9.

Burton, R. S., Ellison, C. K., \& Harrison, J. S. (2006). The sorry state of F-2 hybrids: Consequences of rapid mitochondrial DNA evolution in allopatric populations. The American Naturalist, 168, S14-S24. doi: 10.1038/hdy.2016.31

Civetta, A., \& Gaudreau, C. (2015). Hybrid male sterility between Drosophila willistoni species is caused by male failure to transfer sperm during copulation. BMC Evolutionary Biology, 15, 75-82. doi: 10.1186/s12862-015-0355-8

Coyne, J. A., \& Orr, H. A. (2004). Speciation. Sunderland: Sinauer and Associates.

Coyne, J. A., Elwyn, S., Kim, S. Y., \& Llopart, A. (2004). Genetic studies of two sister species in the Drosophila melanogaster subgroup, D. yakuba and D. santomea. Genetics Research, 84, 11-26.

Davies, N., Aiello, A., Mallet, J., Pomiankoski, A., \& Silberglide, R. E. (1997). Speciation in two neotropical butterflies: Extending Haldane's rule. Proceedings of the Royal Society B: Biological Sciences, 264, 845-851. doi: $10.1017 / \mathrm{S} 0016672304007013$

Dickman, C. T., \& Moehring, A. J. (2013). A novel approach identifying hybrid sterility QTL on the autosomes of Drosophila simulans and D. mauritiana. PLoS One, 8, e73325. doi: 10.1098/rspb.1997.0118

Dobzhansky, T. H. (1937). Genetics and the origin of species. New York: Columbia University Press.

Eads, B. D., Colbourne, J. K., Bohuski, E., \& Andrews, J. (2007). Profiling sex-biased gene expression during parthenogenetic reproduction in Daphnia pulex. BMC Genomics, 8, 464-477. doi: 10.1371/journal.pone.0073325

Futch, D. G. (1966). A study of speciation in South Pacific populations of Drosophila ananassae. University of Texas Publications, 6615, 79-120.

Futch, D. G. (1973). On the ethological differentiation of Drosophila ananassae and D. pallidosa in Samoa. Evolution, 27, 456-467. doi: 10.1186/1471-2164-8-464

Garrigan, D., Kingan, S. B., Geneva, A. J., Vedanayagam, J. P., \& Presgraves, D. C. (2014). Genome diversity and divergence in Drosophila mauritiana: Multiple signatures of faster X evolution. Genome Biology and Evolution, 6, 2444-2458. doi: 10.1093/gbe/evu198

Gomes, S., \& Civetta, A. (2015). Hybrid male sterility and genome-wide misexpression of male reproductive proteases. Scientific Reports, 5, 11976-11987. doi: 10.1038/srep11976

Good, J. M., Dean, M. D., \& Nachman, M. W. (2008). A complex genetic basis to X-linked hybrid male sterility between two species of house mice. Genetics, 179, 2213-2228. doi: 10.1534/genetics.107.085340

Gow, J. L., Peichel, C. L., \& Taylor, E. B. (2007). Ecological selection against hybrids in natural populations of sympatric three spine sticklebacks. Journal of Evolutionary Biology, 20, 2173-2180. doi: $10.1111 / \mathrm{j} .1420-9101.2007 .01427 . \mathrm{x}$

Haerty, W., Jagadeeshan, S., Kulathinal, R. J., Wong, A., Ravi Ram, K., Sirot, L. K., Levesque, L., Artieri, C. G., Wolfner, M. F., Civetta, A., \& Singh, R. S. (2007). Evolution in the fast lane: Rapidly evolving sex-related genes in Drosophila. Genetics, 177, 1321-1335. doi: 10.1534/genetics.107.078865

Haldane, J. B. S. (1922). Sex ratio and unisexual sterility in hybrid animals. Journal of Genetics, 12, 101-109.

Harrison, P. W., Wright, A. E., Zimmer, F., Dean, R., Montgomery, S. H., Pointer, M. A., \& Mank, J. E. (2015). Sexual selection drives evolution and rapid turnover of male gene expression. Proceedings of the National Academy of Sciences, USA, 112, 4393-4398. doi: 10.1073/pnas.1501339112

Hatfield, T., \& Schluter, D. (1999). Ecological speciation in sticklebacks: environment-dependent hybrid fitness. Evolution, 53, 866-873. doi: 10.1111/j.1558-5646.1999.tb05380.x 
Hutter, P. (1997). Genetics of hybrid inviability in Drosophila. Advances in Genetics, 36, 157-185. doi: 10.1016/S0065-2660(08)60309-0

Johnson, N. (2000). Gene interactions and the origin of species. In M. J. Wade (Ed.), Epistasis and the evolutionary process (pp. 197-212). New York: Oxford University Press.

Johnson, N. A., \& Porter, A. H. (2007). Evolution of branched regulatory genetic pathways: Directional selection on pleiotropic loci accelerates developmental system drift. Genetica, 129, 57-70. doi: 10.1007/s10709-006-0033-2

Kaneshiro, K. Y. (1983). Sexual selection and the direction of evolution in the biosystematics of Hawaiin Drosophiladae. Annual Review of Entomology, 28, 161-178. doi: 10.1146/annurev.en.28.010183.001113

Kao, J. Y., Zubair, A., Salomon, M. P., Nuzhdin, S. V., \& Campo, D. (2015). Population genomic analysis uncovers African and European admixture in Drosophila melanogaster populations from the southeastern United States and Caribbean Islands. Molecular Ecology, 24, 1499-1509. doi: 10.1111/mec.13137

Kim, B. K., \& Watanabe, T. K. (1989). Evolutionary genetics of the Drosophila montium subgroup. I. Reproductive isolation and the phylogeny. The Japanese Journal of Genetics, 64, 177-190. doi: 10.1266/jjg.64.177

Kitano, J., Ross, J. A., Mori, S., Kume, M., Jones, F. C., Chan, Y. F., Absher, D. M., Grimwood, J., Schmutz, J., Myers, R. M., Kingsley, D. M., \& Peichel, C. L. (2009). A role for a neo-sex chromosome in stickleback speciation. Nature, 461, 1079-1083. doi: 10.1038/nature08441

Landry, C. R., Hartl, D. L., \& Ranz, J. M. (2007). Genome clashes in hybrids: Insights from gene expression. Heredity, 99, 483-493. doi: 10.1038/sj.hdy.6801045

Malone, J. H., \& Michalak, P. (2008). Physiological sex predicts hybrid sterility regardless of genotype. Science, 319, 59. doi: $10.1126 /$ science. 1148231

Maside, X. R., Barrel, J. P., \& Naveira, H. (1998). Hidden effects of X chromosome introgressions on spermatogenesis in Drosophila simulans x D. mauritiana hybrids unveiled by interactions among minor genetic factors. Genetics, 150, 745-754.

Masly, J. P., \& Presgraves, D. C. (2007). High-resolution genome-wide dissection of the two rules of speciation in Drosophila. PLoS Biology, 5, 1890-1898. doi: 10.1371/journal.pbio.0050243

Mayr, E. (1942). Systematics and the origin of species. New York: Columbia University Press.

Meiklejohn, C. D., Parsch, J., Ranz, J. M., \& Hartl, D. L. (2003). Rapid evolution of male-biased gene expression in Drosophila. Proceedings of the National Academy of Sciences, USA, 100, 9894-9899. doi: 10.1073/pnas.1630690100

Michalak, P., \& Noor, M. A. (2003). Genome-wide patterns of expression in Drosophila pure species and hybrid males. Molecular Biology and Evolution, 20, 1070-1076. doi: 10.1093/molbev/msg119

Michalak, P., \& Noor, M. A. (2004). Association of misexpression with sterility in hybrids of Drosophila simulans and D. mauritiana. Journal of Molecular Evolution, 59, 277-282. doi: 10.1007/s00239-004-2622-y

Mishra, P. K., \& Singh, B. N. (2007). Assessing the putative roles of X-autosome and X-Y interactions in hybrid male sterility of the Drosophila bipectinata species complex. Genome, 50, 653-659. doi: 10.1139/G07-049

Moehring, A. J., Teeter, K. C., \& Noor, M. A. (2007). Genome-wide patterns of expression in Drosophila pure species and hybrid males. II. Examination of multiple-species hybridizations, platforms, and life cycle stages. Molecular Biology and Evolution, 24, 137-145. doi: 10.1093/molbev/msl142

Muller, H. J. (1942). Isolating mechanisms, evolution, and temperature. Biology Symposium, 6, 71-125.

Niehuis, O., Judson, A. K., \& Gadau, J. (2008). Cytonuclear genic incompatibilities cause increased mortality in male F2 hybrids of Nasonia giraulti and $N$. vitripennis. Genetics, 178, 413-426. doi: 10.1534/genetics.107.080523

Noor, M. A. (1997). Genetics of sexual isolation and courtship dysfunction in male hybrids of Drosophila pseudoobscura and D. persimilis. Evolution, 51, 809-815. doi: 10.1111/j.1558-5646.1997.tb03663.x

Oguma, Y. (1993). Sexual behaviour. In Y. N. Tobari (Ed.), Drosophila ananassae: Genetical and biological aspects (pp. 199-207). Tokyo, Japan: Japan Scientific Societies Press. doi: 10.1159/000422637 
Oliveira, D. C., Raychoudhury, R., Lavrov, D. V., \& Werren, J. H. (2008). Rapidly evolving mitochondrial genome and directional selection in mitochondrial genes in the parasitic wasp Nasonia (Hymenoptera: Pteromalidae). Molecular Biology and Evolution, 25, 2167-2180. doi: 10.1093/molbev/msn159

Porter, A. H., \& Johnson, N. A. (2002). Speciation despite gene flow when developmental pathways evolve. Evolution, 56, 2103-2111. doi: 10.1111/j.0014-3820.2002.tb00136.x

Ramsey, J., Bradshaw, H. D., \& Schemske, D. W. (2003). Components of reproductive isolation between the monkeyflowers Mimulus lewisii and M. cardinalis (Phrymaceae). Evolution, 57, 1520-1534. doi: 10.1111/j.0014-3820.2003.tb00360.x

Ranz, J. M., Castillo-Davis, C. I., Meiklejohn, C. D., \& Hartl, D. L. (2003). Sex dependent gene expression and evolution of the Drosophila transcriptome. Science, 300, 1742-1745. doi: 10.1126/science.1085881

Rogers, S. M., \& Bernatchez, L. (2006). The genetic basis of intrinsic and extrinsic post-zygotic reproductive isolation jointly promoting speciation in the lake whitefish species complex (Coregonus clupeaformis). Journal of Evolutionary Biology, 19, 1979-1994. doi: 10.1111/j.1420-9101.2006.01150.x

Rundle, H. D., Nagel, L., Boughman, J. W., \& Schluter, D. (2000). Natural selection and parallel speciation in sympatric sticklebacks. Science, 287, 306-308. doi: 10.1126/science.287.5451.306

Russo, C. A. M., Beatriz, M., Frazão, A., \& Voloch, C. M. (2013). Phylogenetic analysis and a time tree for a large drosophilid data set (Diptera: Drosophilidae). Zoological Journal of the Linnean Society, 169, 765-775. doi: 10.1111/zoj.12062

Sawamura, K., Zhi, H., Setoguchi, K., Yamada, H., Matsuda, M., \& Oguma, Y. (2008). Genetic analysis of female mating recognition between Drosophia ananassae and D. pallidosa: Application of interspecific mosaic genome lines. Genetica, 133, 179-185. doi: 10.1007/s10709-007-9198-6

Schilthuizen, M., Giesbers, M. C. W. G., \& Beukeboom, L. W. (2011). Haldane's rule in the 21st century. Heredity, 107, 95-102. doi: 10.1038/hdy.2010.170

Shapiro, J. A., Huang, W., Zhang, C., Hubisz, M. J., Lu, J., ..., \& Wu, C. I. (2007). Adaptive genic evolution in the Drosophila genomes. Proceedings of the National Academy of Sciences, USA, 104, 2271-2276. doi: 10.1073/pnas.0610385104

Sundararajan, V., \& Civetta, A. (2011). Male sex interspecies divergence and down regulation of expression of spermatogenesis genes in Drosophila sterile hybrids. Journal of Molecular Evolution, 72, 80-89. doi: 10.1007/s00239-010-9404-5

Tao, Y., Xhen, S. N., Hartl, D. L., \& Laurie, C. C. (2003). Genetic dissection of hybrid incompatibilities between Drosophila simulans and D. mauritiana. I. Differential accumulation of hybrid male sterility effects on the X and autosomes. Genetics, 164, 1383-1397.

Turelli, M., \& Orr, H. A. (1995). The dominance theory of Haldane's rule. Genetics, 140, 389-402.

Vishalakshi, C., \& Singh, B. N. (2006). Sexual isolation between two sibling species of Drosophila: D. ananassae and D. pallidosa. Current Science, 90, 1003-1006.

Werren, J. H., Richards, S., Desjardins, C. A., Niehuis, O., Gadau, J., ..., \& Gibbs, R. A. (2010). Functional and evolutionary insights from the genomes of three parasitoid Nasonia species. Science, 327(5963), 343-348.

Wu, C. I., Johnson, N. A., \& Palopoli, M. F. (1996). Haldane's rule and its legacy: Why are there so many sterile males? Trends in Ecology \& Evolution, 11(7), 281-284. doi: 10.1016/0169-5347(96)10033-1

Yukilevich, R., \& True, J. R. (2008). Incipient sexual isolation among cosmopolitan Drosophila melanogaster populations. Evolution, 62(8), 2112-2121. doi: 10.1111/j.1558-5646.2008.00427.x

Zhang, Y., Sturgill, D., Parisi, M., Kumar, S., \& Oliver, B. (2007). Constraint and turnover in sex-biased gene expression in the genus Drosophila. Nature, 450(7167), 233-237. doi: 10.1038/nature06323

Zhang, Z., Hambuch, T. M., \& Parsch, J. (2004). Molecular evolution of sex-biased genes in Drosophila. Molecular Biology and Evolution, 21(11), 2130-2139. doi: 10.1093/molbev/msh223

\section{Copyrights}

Copyright for this article is retained by the author(s), with first publication rights granted to the journal.

This is an open-access article distributed under the terms and conditions of the Creative Commons Attribution license (http://creativecommons.org/licenses/by/4.0/). 